\title{
Teachers' conflicting conceptual models and the efficacy of formative assessments
}

\author{
Gregory D. Kranich ${ }^{1,2}$, Michael C. Wittmann ${ }^{2,3}$ and Carolina Alvarado ${ }^{2}$ \\ ${ }^{1}$ University of Maine Cooperative Extension, State 4-H Office, 5741 Libby Hall, Orono, ME 04469-5741 \\ ${ }^{2}$ University of Maine, Center of Research in STEM Education, 5727 Estabrooke Hall, Orono, ME 04469 \\ ${ }^{3}$ University of Maine, Department of Physics and Astronomy, 5709 Bennett Hall, Orono, ME 04469
}

\begin{abstract}
We studied a group of middle school teachers as they modified curriculum and developed common formative assessments on force and motion concepts. While designing an item and discussing goals for student understanding of acceleration, two of the teachers held opposing models (one of them being incomplete) about the implications of the sign of acceleration on the direction of an object's motion and whether it was speeding up or slowing down. Failing to resolve the inconsistency between their individual models, the teachers wrote an assessment item for which both models would provide the same correct response, albeit for different reasons. The potential to elicit correct answers for incorrect reasons created ambiguity in the ability to recognize probable alternative conceptions. More specifically, the item had limited ability both to refine the teachers' own conceptual understanding and to accurately inform their instruction, interventions, and feedback that would support students in identifying their mistakes.
\end{abstract}

PACS: 01.40.G-, 01.40.-d, 01.40.J-

\section{INTRODUCTION}

When teachers are asked to write assessments of their students' knowledge, one goal is to understand the models students have about a given topic. This diagnosis allows a teacher to adjust subsequent instruction in order to best meet the needs of the learner as the learning process occurs.

Studies have shown that the creation of this feedback loop has a significant positive impact on student performance in science education $[1,2,3]$. The practice of formative assessment has, however, been subject to scrutiny in its implementation, reported effectiveness, and its lack of attention to conceptual detail $[4,5,6,7]$.

For the formative assessment cycle to be most effective, teachers must be able to ask the right kind of question in order to elicit the desired information. Next, they must be able to interpret those data in a manner that accurately represents both a student's current understanding and the nature of alternative conceptions. Finally, in light of this interpretation as evidence, teachers must be able to provide feedback and appropriate interventions that are best suited to that particular learner [1].

This requires a complex network of knowledge that includes, but is certainly not limited to, science content and knowledge for assessment [8]. In this paper we present a case in which teachers' incomplete content knowledge marginalized the efficacy of a formative assessment item on accelerated motion they were designing as a group.

\section{KINEMATICS CONCEPTS}

Student and teacher difficulties in kinematics have been well documented at all levels of instruction [9,10,11]. This research has contributed to a better understanding of student ideas and informed curriculum reform aimed at improved student learning. Students have consistently demonstrated an inability to transfer the learned formalism to analyses of motion that has been observed or described [9]. For the purposes of this paper, we focus specifically on acceleration in one dimension.

\section{RESEARCH SETTING}

Over the span of two weeks in August of 2014, a selfselected group of four middle school science teachers (grades seven and eight) worked with one author (GDK) as a facilitator to produce a sequence of instructional activities. Motivation for this work arose from a general dissatisfaction with the common curriculum used in previous years by the four teachers and a larger cohort of their peers. The four teachers would begin piloting their modifications in September and October of the upcoming school year. These activities focused on the concepts of forces and motion. Within the group, the number of years of teaching experience ranged from less than five years to more than twenty, with various levels of specialization in physical and/or life science.

This study centers on the team's continuation of this curriculum project during one of four monthly meetings in which they created an assessment to accompany a module on accelerated motion. Data were gathered by videotaping group interactions as teachers discussed student learning goals and designed assessment items.

During group discourse, contrasting conceptual understanding occasionally surfaced, and was typically resolved. In this paper, we discuss a case of model inconsistency between two teachers and the assessment item developed by the cohort that did not discriminate between the two teachers' models.

\section{CONTRASTING MODELS}

While considering the context of non-uniform motion and concepts warranting assessment, the group was unanimous in their support of "positive" and "negative 
acceleration." Both the presence of the these terms in the textbook common to their collective classrooms and the call for students' ability to apply Newton's laws of motion per the Next Generation Science Standards (NGSS) provided impetus for the decision [12].

Though assessing student knowledge of positive and negative acceleration had been identified as a target by the teachers, no one had made explicit what the expected students' understanding was and what might constitute acceptable evidence of such. Pushing the teachers to clarify their thinking, the group facilitator (author GDK) asked what they felt was important for students to know and be able to do. During the discussion that followed, John and Derrick (aliases) argued for two different ways of thinking about the sign of the acceleration.

\section{A. "Speeding up is positive acceleration" model}

John argued to say that speeding up is always positive acceleration, and slowing down is always negative, regardless of the direction (forward or backwards) an object is moving at the time. To illustrate, he offered the following example:

John: Let's say you put a big fan at the end; the car's coming toward it. It stops; it goes the other way. That has to be a positive acceleration, because it was speeding up going the opposite direction. It can't be a negative acceleration. Negative acceleration would be the slowing down to the stop, and then a positive-If it's speeding up going in the opposite direction, wouldn't that be positivestill positive acceleration; it's getting faster. I've always seen that positive acceleration is an increase...

John's description is consistent with the idea that acceleration is an indicator of changing speed. More specifically, positive acceleration always describes an increase in speed, and negative acceleration a decrease, independent of the direction of motion. This reasoning correctly indicates the sign of acceleration when speeding up or slowing down with a positive velocity, but generates incorrect choices when moving with a negative velocity.

This view is not like previously identified examples of students confusing acceleration and velocity, described as "nondifferentiated protoconcepts" by Trowbridge and McDermott [9, p.245]. Instead, John's model considers the direction of motion to be irrelevant, as his words make clear. We argue that this view does not consider acceleration to be a vector quantity without explicit reference to the direction of acceleration. Instead, the model interprets "positive" and "negative" to indicate "increase" and "decrease," as aligned with a more colloquial usage of the words. John used directional hand gestures while talking about "towards the fan" and "the opposite direction" to describe the direction of velocity, but showed no signs of direction when talking about "positive" and "negative." This suggests that he was not conflating the two concepts.

\section{B. "Directions are Independent" Model}

In contrast to John, Derrick paid particular attention to the direction of the acceleration and its relationship to both the change in magnitude of speed and the direction of motion. Derrick tried unsuccessfully to make the point that isolated knowledge of the direction of acceleration indicates neither the direction of motion nor the change in speed. He saw this as a potentially problematic idea for students. His speech was broken and full of starts and stops as he tried to communicate his thinking to the group. To better convey Derrick's struggle to articulate his ideas, we have chosen to present the quotations verbatim.

Derrick: ... and I'd start thinking about how would they [students] misunderstand negative acceleration. One, I would tell them - kid might think you are accelerating in the opposite direction - but you are, but that you might be actually moving, you know. The whole idea of, like, 'net forces equal motion' - I know we aren't talking about forces yet - but you know, so if it's negative acceleration, does that mean that am I actually going in the direction? Does motion happen in the direction of the acceleration?

We see Derrick trying to use his knowledge of dynamics and his awareness of common student difficulties to think about the situation, but his words are jumbled and hard to understand. Later, he tried again:

Derrick: I just wonder if someone might say, "Well negative acceleration...that must mean that instead of like positive acceleration, that negative is moving backwards, like a [negative] change in position. So would negative acceleration be a change in position, I mean it could be, but it could also be slowing down [in the positive direction]."

Derrick was trying to be clear about the confusions that may arise, namely between the direction of acceleration for the direction of velocity. Throughout, Derrick was struggling to convey the idea that direction and sign could be different for velocity and acceleration:

Derrick: ... [the] problem is that you coul-I mean it means, it doesn't necce-it means you're accelerating in that direction, but it doesn't mean that you're moving in that direction. See what I'm saying?

The group did not respond strongly to these ideas.

\section{C. "Using vectors" approach}

After both Derrick and John had presented their ideas, the facilitator (author GDK) tried to help resolve the differences. Using a whiteboard and marker and representations of vectors (arrows) that were in common use by the group at other points of their instruction, he suggested the correct answer, with velocity and acceleration arrows that might point in the same direction or different directions. These were accompanied by words 
about positive and negative directions of motion and acceleration, as well. This attempt was unsuccessful in changing John's mind or clarifying Derrick's points for the group.

By the end of the conversation, teachers were aware that positive acceleration does not necessarily mean an increase in speed. This was elucidated by John's question: "Can you have a negative acceleration speeding up?" to which the response was, "yes." Nevertheless, John did not change his mind, speaking to the persistence of the individuals' views of the concept.

\section{Contrasting Domains}

Faced with a nontrivial conceptual conflict and time constraints, the group accepted that, "As long as an object is moving in the positive direction, positive acceleration is speeding up, and negative acceleration is slowing down." This agreed upon model, while correct, is however, incomplete.

In Table 1, we present how someone using the two models would determine the sign of the acceleration for a variety of situations. We note that the answers agree for positive velocity, and disagree for negative velocity. We explore the consequences of this decision on the teachers' assessment.

\section{AN ASSESSMENT THAT DOES NOT PROVIDE CONTRASTS}

After the conversation between John and Derrick, the teachers created the assessment item shown in Fig. 1. The assessment was created outside of group discussion and we are not aware of how it was created. Instead, we evaluate its content and what teachers might learn from it.

The question context is of cars moving in what is shown as the positive direction, with one car slowing down. We note that parts $i$ and iii of the question are consistent with the overall goals of instruction. We analyze part ii because it shows the consequences of the unresolved conflict in John and Derrick's models of accelerated motion, in particular the meanings of positive and negative

TABLE 1. Signs of acceleration in different situations, according to models described by John and Derrick.

\begin{tabular}{c|c|c}
\hline Situation & $\begin{array}{c}\text { Speeding Up }= \\
\text { Positive Accel. }\end{array}$ & $\begin{array}{c}\text { Directions are } \\
\text { Independent }\end{array}$ \\
\hline $\begin{array}{l}v \text { positive, } \\
\text { increasing }\end{array}$ & $a>0$ & $a>0$ \\
\hline $\begin{array}{l}v \text { positive, } \\
\text { decreasing }\end{array}$ & $a<0$ & $a<0$ \\
\hline $\begin{array}{l}v \text { negative, } \\
\text { increasing }\end{array}$ & $a>0$ & $a<0$ \\
\hline $\begin{array}{l}v \text { negative, } \\
\text { decreasing }\end{array}$ & $a<0$ & $a>0$ \\
\hline
\end{tabular}

Item 4. The image below represents two cars riding next to each other. A picture is taken every second to show the cars' position. The number line represents the position in meters. Use the image to answer the questions that follow.

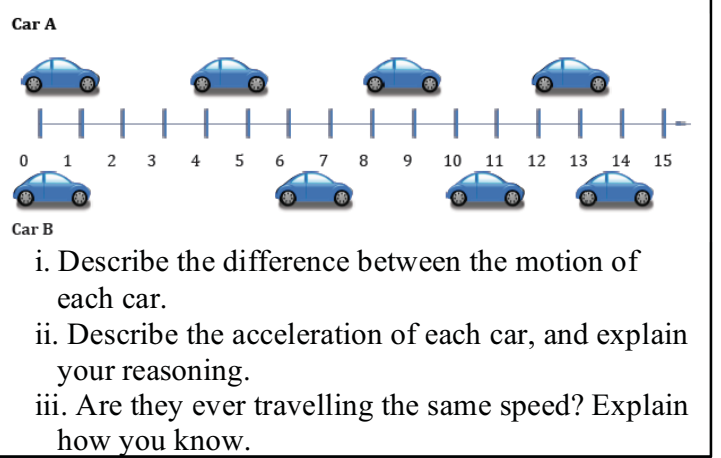

FIG 1. Assessment item created by teachers to assess student knowledge of acceleration.

acceleration.

If we consider responses based on John and Derrick's models, we arrive at possible responses like those shown in Table 2. Both models lead to a response in which Car B is considered to have a negative acceleration. Though the answer based on Derrick's model includes a description of the direction as part of the explanation, and John's does not, the answers are virtually the same.

If we were only depending on these written responses, it is likely that a student would be considered correct, regardless of their model. As a result, the student would not receive adequate feedback or intervention in order to recognize the limitations of this model. Thus, the question as asked might lead teachers to believe that these students possessed sufficient understanding of the topic, when some might actually have the same confusion that John had.

In sum, we see the integral feedback loop of formative assessment disrupted by the lack of appropriate resolution of the conflict existing in John's model. More specifically, the question does not elicit sufficient evidence of a particular difficulty, which would lead to an inaccurate interpretation not uncovering the need for corrective feedback and adjusted instruction. Although these specific limitations were not discussed by the group, they were aware that the simplified model agreed upon in the previous section could only be true in one direction.

As an aside, we suggest that a teacher wishing to

TABLE 2. Inferred Responses to Assessment Item 4.ii.

\begin{tabular}{c|c|c}
\hline Model & $\begin{array}{c}\text { Speeding Up } \\
\text { Positive Accel. }\end{array}$ & $\begin{array}{c}\text { Directions are } \\
\text { Independent }\end{array}$ \\
\hline \multirow{2}{*}{$\begin{array}{c}\text { Inferred } \\
\text { Response }\end{array}$} & $\begin{array}{c}\text { negative } \\
\text { acceleration } \\
\text { because it is } \\
\text { slowing down. }\end{array}$ & $\begin{array}{c}\text { Car B has a negative } \\
\text { acceleration because it } \\
\text { is slowing down in the } \\
\text { positive direction. }\end{array}$ \\
\hline
\end{tabular}


distinguish among students who have the two models presented by John and Derrick would have to create an assessment item that used negative velocity as its context. As shown, a positive velocity question would not indicate a student's individual model of acceleration. We credit our ability to achieve understandings of both models extending beyond their likely item response to the attempts made by John and Derrick to share the details of their thinking with the group. Thus, we are able to assert that their reasoning is, in fact, dissimilar.

\section{CONCLUSIONS}

Acceleration remains a difficult concept for students to master even at the college level $[9,10,11]$, and we observe teachers also struggling in this area. A complete understanding of the topic requires a solid grasp of vectors, coordinate systems, displacement, and velocity, ideas that are not often fully developed in the science classroom $[9,10,11]$. Thus, it is critical to provide students with guidance as they develop an understanding of these abstract concepts. Time constraints compound the difficulties in helping students achieve mastery.

In our study, teachers designed an item that was successful in providing insight into a student's understanding of uniform versus non-uniform motion. However, despite the fact that the primary goal was to assess student understanding of positive and negative acceleration, the item investigated in this study lacked the ability to discern between two models of acceleration as

[1] P. Black and D. Wiliam, Assessment and Classroom Learning (1998).

[2] P. Black, C. Harrison, C. Lee, B. Marshall, and D. Wiliam, Phi Delta Kappan 86, 8 (2004).

[3] B. Cowie and B. Bell, Assess. Educ. Princ. Policy Pract. 6, 101 (1999).

[4] J. E. Coffey et al., J. Res. Sci. Teach. 48, 1109 (2011).

[5] R.E. Bennett, Assess. Educ. Princ. Policy Pract. 18, 5 (2011).

[6] D. Wiliam, Educ. Assess. 11, 283 (2006).

[7] V.K. Otero, J. Teach. Educ. 57, 247 (2006). described by two different teachers. We attribute this limitation to an incomplete conceptual understanding held by the teachers.

The potential for students to provide a correct answer for the wrong reason disrupts the feedback loop between teachers and students meant to providing learning opportunities to meet students' needs. These false positive responses promote an invalid interpretation of student performance that suggests an inflated sense of mastery and does not allow teachers to accurately resolve a student's particular conceptual model. Thus, teachers would neither be able to provide adequate feedback to students, nor could they use response data to inform successive instruction based on a specific conceptual difficulty. This limitation undermined the utility of the item as a tool for formative assessment to ascertain and respond to student ideas during the process of developing an understanding of acceleration.

\section{ACKNOWLEDGMENTS}

We thank Alex Axthelm, Oai Ha, and Laura Millay for their help with this research. We also thank the teachers of the Maine Physical Sciences Partnership who participated in the activity. We wish to keep in mind that this paper only represents a very small portion of the tremendous work and effort that the teachers involved devoted to improving physics instruction in middle school classrooms. This work was supported in part by NSF grants MSP-0962805 and DRL-1222580.

[8] S. Avargil, O. Herscovitz, and Y.J. Dori, J. Sci. Educ. Technol. 21, 207 (2011).

[9] D.E. Trowbridge and L. McDermott, Am. J. Phys. 49, 242 (1981).

[10] P.S. Shaffer and L.C. McDermott, Am. J. Phys. 73, 921 (2005).

[11] I.A. Halloun and D. Hestenes, Am. J. Phys. 53, 1056 (1985).

[12] NGSS Lead States, Achieve, Inc. Behalf Twenty-Six States Partners That Collab. NGSS 1 (2013). 